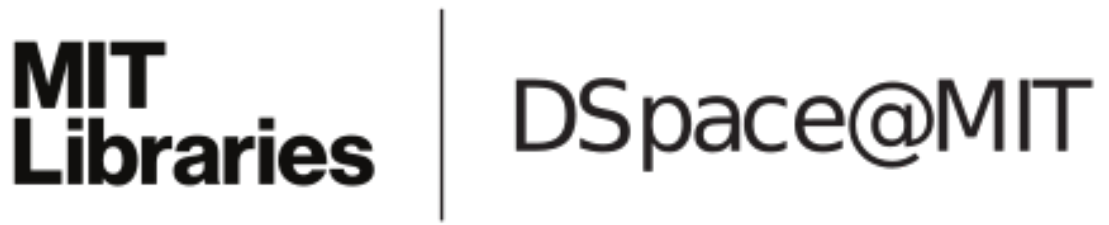

\author{
MIT Open Access Articles
}

\section{Optical Generation of Gigahertz-Frequency Shear Acoustic Waves in Liquid Glycerol}

The MIT Faculty has made this article openly available. Please share how this access benefits you. Your story matters.

Citation: Pezeril, T. et al. “Optical Generation of Gigahertz-Frequency Shear Acoustic Waves in Liquid Glycerol.” Physical Review Letters 102.10 (2009): 107402. (C) 2010 The American Physical Society.

As Published: http://dx.doi.org/10.1103/PhysRevLett.102.107402

Publisher: American Physical Society

Persistent URL: http://hdl.handle.net/1721.1/51368

Version: Final published version: final published article, as it appeared in a journal, conference proceedings, or other formally published context

Terms of Use: Article is made available in accordance with the publisher's policy and may be subject to US copyright law. Please refer to the publisher's site for terms of use. 


\title{
Optical Generation of Gigahertz-Frequency Shear Acoustic Waves in Liquid Glycerol
}

\author{
T. Pezeril, ${ }^{1, *}$ C. Klieber, ${ }^{1}$ S. Andrieu, ${ }^{2}$ and K. A. Nelson ${ }^{1}$ \\ ${ }^{1}$ Department of Chemistry, Massachusetts Institute of Technology, Cambridge, Massachusetts 02139, USA \\ ${ }^{2}$ LPM UMR-CNRS 7556, Université H. Poincaré, 54506 Vandoeuvre, France
}

(Received 16 September 2008; published 10 March 2009)

\begin{abstract}
Picosecond laser ultrasonic techniques for acoustic wave generation and detection have been employed to probe shear acoustic waves in liquid glycerol at gigahertz frequencies. The experimental approach uses a unique laser pulse shaping technique and a crystallographically canted metal layer to generate frequency-tunable transverse acoustic waves, and uses time-domain coherent Brillouin scattering to detect the waves after they propagate through a liquid layer and into a solid substrate. A linear frequency dependence is found for both the shear speed and attenuation rate in glycerol.
\end{abstract}

DOI: 10.1103/PhysRevLett.102.107402

Fast structural relaxation dynamics in liquids continue to pose major fundamental challenges [1], in large measure because direct experimental access to key relaxing degrees of freedom over the time or frequency ranges of interest remains elusive. Both density and shear relaxation play central roles in the complex structural responses of viscoelastic materials. On slow time scales, dynamic mechanical analysis and sonic or related measurement methods can be used, while faster responses require measurements of longitudinal and shear acoustic waves in the megahertz and gigahertz frequency ranges. Much of the $\mathrm{MHz}$ range is now accessible to ultrasonics and impulsive stimulated thermal or Brillouin scattering (impulsive stimulated thermal or Brillouin scattering [2-5]), and (usually isolated) frequencies in the low GHz range may be accessed through spontaneous Brillouin scattering [6]. Recent work in x-ray Brillouin scattering has accessed $\mathrm{THz}$ longitudinal acoustic frequency ranges [7], but frequencies in the tens to hundreds of $\mathrm{GHz}$ range, where fast relaxation features occur, have remained difficult to access. Deep-UV Brillouin scattering from longitudinal acoustic waves in this range has been demonstrated [4,7], but its utility is limited by strong absorption in most materials. Picosecond ultrasonics [8], in which a short optical pulse generates a single-cycle acoustic pulse that is observed after propagation through a sample, has provided tabletop access to much of the GHz-frequency range for longitudinal acoustic waves. Adaptations of the method to enable $\mathrm{GHz}$ shear wave generation [9-12] have been developed. However, shear waves in liquids have remained elusive, to the extent that the challenges in "seeking shear waves in liquids with picoseconds ultrasonics" [13] have been elaborated explicitly. The use of multiple optical pulses to generate frequency-tunable, multiple-cycle longitudinal waves [14] has been demonstrated to improve acoustic spectral brightness for characterization of frequency-dependent material responses. Here we demonstrate this approach for generation of frequency-tunable shear as well as longitudinal acoustic waves in the GHz-frequency range. We further demonstrate a sample and optical configuration that
PACS numbers: 78.20.Hp, 43.35.+d, 78.40.Dw, 78.47.J-

permit the measurements to be conducted in viscoelastic liquids, whose $\mathrm{GHz}$-frequency acoustic responses are of particular interest in connection with complex structural relaxation dynamics. We present results from glycerol liquid at room temperature, in which shear acoustic waves in the 18-50 GHz range and longitudinal acoustic waves in the 30-90 GHz range have been characterized. Our approach opens the door to versatile shear and longitudinal acoustic spectroscopy of bulk materials and of nanometer solid and liquid layers down to monolayer thicknesses.

In the present front-back optical pump-probe type measurement (see Fig. 1), longitudinal and shear acoustic waves were optically generated in an iron semitransparent thin film upon ultrafast laser irradiation. The iron films of about $50 \mathrm{~nm}$ thickness were obliquely deposited under

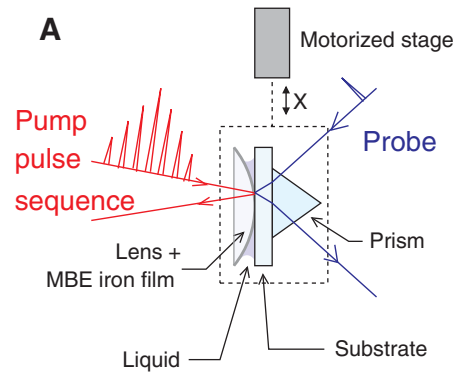

B

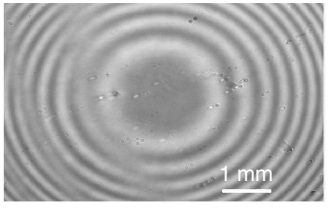

FIG. 1 (color online). (A) The liquid under study is squeezed between a transparent flat substrate and a plano-convex lens. The curved side of the lens is coated with an iron thin film with canted crystallographic orientation. A sequence of seven optical excitation pulses generates multiple-cycle acoustic waves in the iron film. After propagation through the liquid layer, the acoustic waves are detected in the transparent solid substrate by timeresolved coherent Brillouin scattering of variably delayed $400 \mathrm{~nm}$ probe pulses. The pulse sequence timing is adjusted to match the Brillouin scattering frequency for the optimal signal level. Data are collected at several lateral $(X)$ positions of the sample, corresponding to several different liquid layer thicknesses. (B) Newton's ring interference pattern from the sample. The lens curvature of $2.5 \mathrm{~m}$ yields the thickness of the liquid film at a given $X$ position. 
ultrahigh vacuum [15], with an oblique incidence angle of $60^{\circ}$, providing a tilt of the crystal symmetry axis of about $40^{\circ}$. The break of the shear symmetry in the direction normal to the sample ensures direct thermoelastic generation of shear acoustic plane waves [11], in addition to longitudinal acoustic plane waves that do not require such a canted crystallographic orientation to be excited. The excitation pulses from a Ti:Sapphire amplifier system (Coherent RegA) operating at a $250 \mathrm{kHz}$ repetition rate were centered around $790 \mathrm{~nm}$ with a bandwidth of about $8 \mathrm{~nm}$ and a pulse duration of $200 \mathrm{fsec}$. They were focused on the sample to a $100 \mu \mathrm{m}$ spot with an average pulse energy of $1 \mu \mathrm{J}$. Both longitudinal and shear acoustic waves may propagate into an adjacent liquid layer, and may be detected after transmission into a transparent substrate via time-domain coherent Brillouin light scattering $[8,16]$. The coherently scattered field, whose optical phase varies depending on the acoustic wave peak and null positions, superposes with the reflected probe field, resulting in signal intensity that shows time-dependent oscillations at the acoustic frequency (see Fig. 2). This frequency $\nu$ is determined as in any Brillouin scattering measurement by the probe wavelength $\lambda$, the scattering angle $\theta$, the refractive index $n$, and the acoustic speed $v$ in the substrate through the relation $\nu=2 n v \sin (\theta / 2) / \lambda$. In the present case, the probe wavelength was fixed at $395 \mathrm{~nm}$ (focused to a $20 \mu \mathrm{m}$ spot size) and either silica glass or sapphire was used for the substrate to provide access to lower or higher
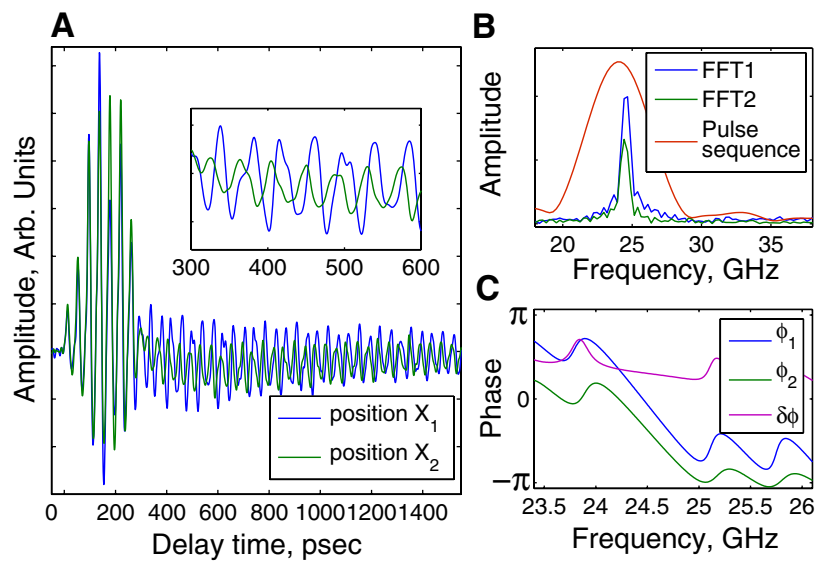

FIG. 2 (color online). (A) Time derivative of the measured signal intensity for two distinct positions $X_{1}$ and $X_{2}$ of the sample corresponding to two distinct liquid layer thicknesses, $d_{1}$ and $d_{2}>d_{1}$. The excitation pulse sequence is visible between 0 and 280 psec due to the electronic response that is induced in the iron film, which is detected by the probe light. This signal is followed by oscillations due to coherent shear waves in the glass substrate. (B) Acoustic amplitude spectra of both acoustic signals, $\mathrm{FFT}_{1}$ and $\mathrm{FFT}_{2}$, and (C) the corresponding phases, $\phi_{1}$ and $\phi_{2}$. The phase and amplitude differences yield the acoustic speed and attenuation length, respectively, in the liquid at the specified Brillouin frequency. Note that in (C), only the phase at frequencies around the Brillouin peak at $24.6 \mathrm{GHz}$ contains relevant information. frequency ranges, respectively. The detection of shear waves is optimized when the probe polarization and the shear acoustic wave polarization are both perpendicular to the plane of incidence, and a crossed polarizer is placed in front of the detector to detect the rotation of probe polarization [17]. The longitudinal wave detection does not require any polarizer. The acoustic frequency that is excited may be varied by changing the excitation pulse sequence timing, and this may be adjusted to match the Brillouin scattering frequency to optimize the acoustic spectral brightness at that frequency. In all cases, measurements were made at multiple lateral positions [ $X$ in Fig. 1(a)] corresponding to multiple liquid layer thicknesses. Differences in Brillouin signal amplitudes from acoustic waves that propagated through different liquid thicknesses yielded acoustic attenuation lengths. Differences in acoustic transit times across liquid layers with thickness differences that were smaller than the $20-50 \mathrm{~nm}$ acoustic wavelengths in the liquid were observed as phase shifts in the time-dependent Brillouin signal oscillations, and yielded the acoustic speed of the liquid. The detected probe light undergoes time-dependent intensity modulation $\delta I$,

$$
\delta I=A \cos (2 \pi \nu t+\phi),
$$

where

$$
A \propto \exp (-\Gamma d) \text { and } \phi \propto-2 \pi \nu(d / v)
$$

at the Brillouin frequency $\nu$ of the substrate material. Here, the Brillouin phase shift $\phi$ is proportional to the traveling time of the acoustic wave through the liquid of thickness $d$ at the acoustic speed $v$. The Brillouin scattering amplitude $A$ is given by the sample thickness and the attenuation coefficient $\Gamma$. In our analysis, we have also taken into account the optical phase shift of the reflected probe light due to the variation in liquid thickness [see Fig. 1(b)], but the acoustic phase shift $\phi$ of Eq. (1) is predominant since the acoustic wavelength is roughly $100 \times$ shorter than the optical wavelength. Measurement of the acoustic phase shift in the data yields subnanometer sensitivity to sample thickness variation, on the order of a single molecular layer. See, for example, the Fig. 3(c) inset.

Our measurements of glycerol acoustic properties were conducted with four different sample configurations: glass substrate and sapphire substrate, each with and without a silica glass prism attached. The glass and sapphire substrates were used to access lower and higher Brillouin frequency ranges, respectively, based on the higher sound speed in sapphire. The prism was attached in order to facilitate variable Brillouin scattering angles in the range of approximately $120^{\circ}-160^{\circ}$ in the substrate, while the substrate without a prism was used to reach larger scattering angles up to the $180^{\circ}$ backscattering limit. Excitation pulse sequences were used as described above to generate multiple-cycle acoustic waves except in measurements of longitudinal waves in a glass substrate, since the signal at the selected Brillouin scattering frequency from this ma- 


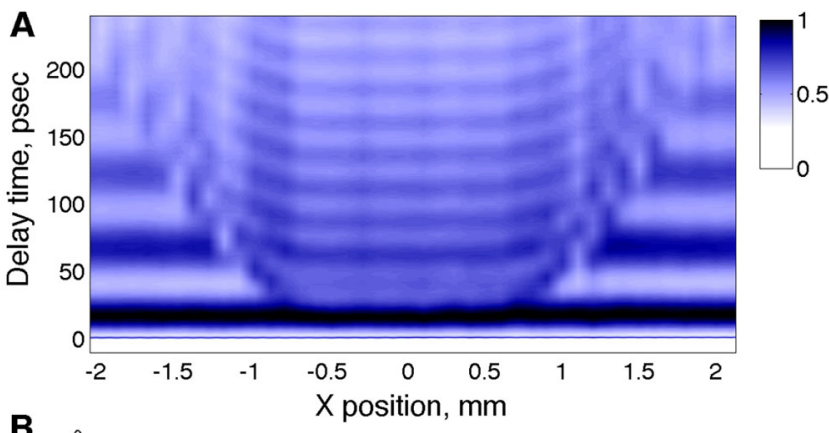

as shown in Figs. 2 and 3, using the ultrafast electronic signal from the iron thin film as an amplitude and time reference as depicted in Fig. 2(a) for an excitation pulse sequence and in Fig. 3(a) for a single excitation pulse. According to formula (2) and the known $2.5 \mathrm{~m}$ radius of curvature of the lens, the measured phase shifts [Fig. 3(c)] and amplitudes [Fig. 3(b)] determined through Fourier analysis of the substrate acoustic wave signals were used to determine the acoustic speed and attenuation coefficient of the liquid at the selected Brillouin frequency. The entire set of phase shift results from multiple liquid thicknesses shown in Fig. 3(c) is used to determine a single quantity, the sound speed at one acoustic frequency, and the entire set of amplitude results shown in Fig. 3(b) is used to determine the attenuation coefficient at the same frequency, resulting in high precision for the values determined. Extensive measurements showed that the variation of liquid layer thickness across different lateral sample positions was determined with a precision of approximately $0.5 \mathrm{~nm}$, that optical cavity effects [evidenced by the Newton's ring pattern in Fig. 1(b)] on the acoustic amplitude measurements were minimized by referencing the level of the acoustic signal to that of the electronic signal for each measurement, and that acoustic cavity effects were negligible.

Results from shear and longitudinal wave measurements in glycerol are shown in Fig. 4. Measurements conducted with multiple scattering angles yielded longitudinal acoustic frequencies in the 31-43 and 57-91 GHz ranges with glass and sapphire substrates, respectively, and corresponding shear acoustic frequencies in the 19-26 and $31-50 \mathrm{GHz}$ ranges. For these preliminary measurements, glycerol was used as purchased, without further purification to eliminate water impurities, and sample temperature

tering from the acoustic waves in liquid glycerol. Near the center of the sample $(X \approx 0)$ the lens and the substrate are almost in direct contact and signal oscillations due to acoustic propagation in the substrate begin promptly. Away from the center of the sample $(|X|>0.5 \mathrm{~mm})$ the transit time through the liquid layer is apparent as a delay in the start of substrate acoustic propagation and a phase shift in the signal oscillations relative to the phase at the center of the sample. Still farther from the center $(|X|>1 \mathrm{~mm})$ the signal oscillations due to acoustic wave propagation in the substrate are further delayed and phase shifted, and these oscillations are preceded by lower-frequency oscillations due to acoustic wave propagation in the liquid. (B) Normalized amplitude values and (C) phase shifts of the $40 \mathrm{GHz}$ highfrequency signal oscillations shown in (A) due to coherent Brillouin scattering from the substrate. Shear wave measurements also were conducted at the same lateral positions $X$ on each sample. (C) The inset illustrates that at the highest sets of longitudinal $(90 \mathrm{GHz})$ and shear $(50 \mathrm{GHz})$ frequencies, the measurements yielded consistent values for liquid layer thickness changes across the sample, with subnanometer sensitivity.

terial was strong even with a single excitation pulse. For each sample configuration and scattering angle, timeresolved longitudinal and shear wave Brillouin scattering data were recorded at different $X$ positions on the sample,

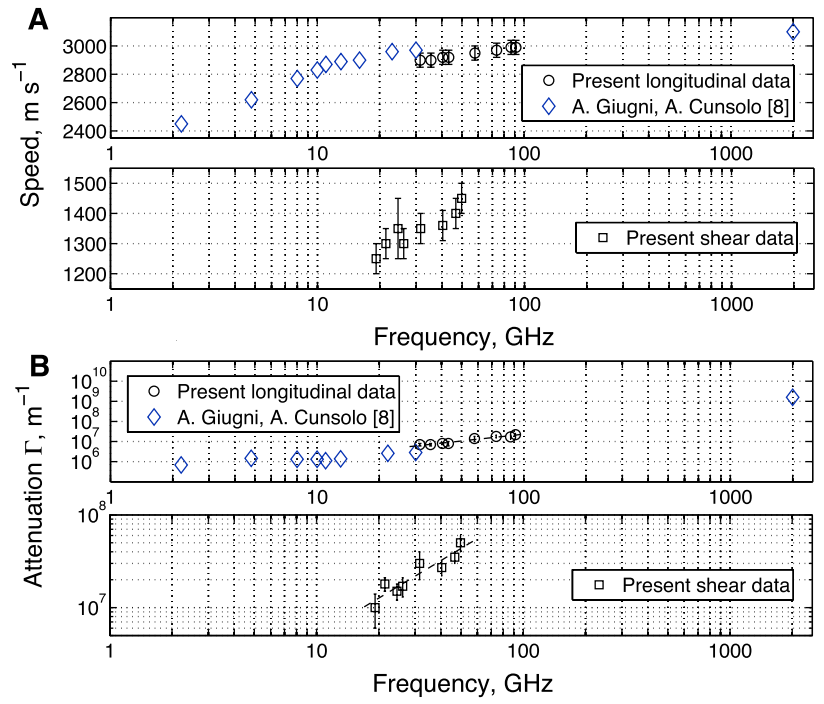

FIG. 4 (color online). Shear and longitudinal acoustic speeds (A) and attenuation coefficients (B) in liquid glycerol at room temperature, from measurements with multiple Brillouin scattering angles using glass and sapphire substrates. 
in the room was not regulated to better than $\pm 2 \mathrm{~K}$. The shear speed of sound in glycerol at room temperature increases approximately linearly with frequency, while the longitudinal speed of sound appears to remain almost constant over the frequency range of the measurements. This suggests that the longitudinal sound speed has reached its infinite-frequency value, relative to the slow, strongly frequency-dependent "alpha" relaxation dynamics [1] that describe the liquid viscoelastic behavior at this temperature. It also suggests that the present frequency range lies below the weakly temperature-dependent, high-frequency (beta) relaxation feature in glycerol. This is consistent with dielectric measurement results [18] that extend to terahertz frequency ranges. The present frequency range is roughly in between the slower (alpha) relaxation dynamics at room temperature and the faster (beta) relaxation dynamics. For the somewhat lower-frequency range of the shear acoustic measurements, the results suggest that the infinitefrequency speed has not yet been reached. This may indicate that the shear acoustic frequency range overlaps with the high-frequency edge of the alpha relaxation spectrum at room temperature. It also may indicate the highfrequency edge of an additional "constant relaxation" feature that has been suggested in glycerol in the 1-10 GHz range [7,19-22]. The shear and longitudinal attenuation coefficients increase with frequency, but with a dependence that appears to be closer to linear than quadratic [23,24]. Phenomenologically, this indicates that the shear viscosity $\eta$ that is linked to the shear attenuation coefficient through the relation [23], $\eta=$ $3 \Gamma_{S} \rho v_{S}^{3} / 2(2 \pi \nu)^{2}$, where $\rho$ is the density, is a decreasing function of frequency in the ranges explored here. This expression of the viscosity is valid when the acoustic wave vector $q_{S}=2 \pi \nu / v_{S}$ exceeds the attenuation coefficient $\Gamma_{S}$, i.e., the acoustic wave is underdamped. In the present case, $q / \Gamma_{S} \approx 6.5$ on average over the frequency range explored. The mean calculated value for the viscosity is $2.9 \mathrm{~m} \mathrm{~Pa}-\mathrm{s}$, comparable to the static viscosity of water and $1000 \times$ smaller than the static glycerol value at room temperature [25], further illustrating that the present shear frequency range lies on the high-frequency wing of the alpha feature of the shear relaxation spectrum of glycerol at this temperature.

A full temperature-dependent study is under way to provide a deeper understanding of the underlying structural relaxation dynamics in glycerol. The frequency ranges that are accessible to the current method could be increased substantially by using 267 or $200 \mathrm{~nm}$ probe light (i.e., third or fourth harmonic of the $800 \mathrm{~nm}$ laser wavelength) and the sapphire substrate and by using a silicon substrate [16]. Parallel efforts are under way to connect the frequency ranges of the present measurements with those of impulsive stimulated scattering measurements at $\mathrm{MHz}$ [26] to low $\mathrm{GHz}$ frequencies. In this manner, a shear acoustic frequency range of about five decades can be achieved. Finally, the approach used here may be applied to ex- tremely thin liquid layers, down to a monolayer, to explore confinement and interfacial effects [27-29].

This work was partially supported by the Department of Energy Grant No. DE-FG02-00ER15087 and the National Science Foundation Grants No. CHE-0616939 and No. DMR-0414895. The authors would like to thank Vitaly Gusev for fruitful discussions.

*pezeril@mit.edu

[1] E. Donth, The Glass Transition: Relaxation Dynamics in Liquids and Disordered Materials (Springer, New York, 2001).

[2] Y. Yan, L. Cheng, and K. A. Nelson, J. Chem. Phys. 88, 6477 (1988).

[3] S. M. Silence, A. R. Duggal, L. Dhar, and K. A. Nelson, J. Chem. Phys. 96, 5448 (1992).

[4] Y. Yang and K. A. Nelson, J. Chem. Phys. 103, 7722 (1995); J. Chem. Phys. 103, 7732 (1995).

[5] D. Paolucci and K. A. Nelson, J. Chem. Phys. 112, 6725 (2000).

[6] D. Pinnow, S. Candau, J. LaMacchia, and T. Litovitz, J. Acoust. Soc. Am. 43, 131 (1968).

[7] A. Giugni and A. Cunsolo, J. Phys. Condens. Matter 18, 889 (2006).

[8] C. Thomsen, H. T. Grahn, H. J. Maris, and J. Tauc, Phys. Rev. B 34, 4129 (1986).

[9] O. Matsuda, O. B. Wright, D. H. Hurley, V. E. Gusev, and K. Shimizu, Phys. Rev. Lett. 93, 095501 (2004).

[10] T. Pezeril et al., Phys. Rev. B 73, 132301 (2006).

[11] T. Pezeril et al., Phys. Rev. B 75, 174307 (2007).

[12] T. Pezeril, F. Leon, D. Chateigner, S. Kooi, and K. A. Nelson, Appl. Phys. Lett. 92, 061908 (2008).

[13] M. Msall, O. B. Wright, and O. Matsuda, J. Phys. Conf. Ser. 92, 012026 (2007).

[14] J. D. Choi, T. Feurer, M. Yamaguchi, B. Paxton, and K. A. Nelson, Appl. Phys. Lett. 87, 081907 (2005).

[15] P. Fréchard et al., Thin Solid Films 263, 42 (1995).

[16] A. Devos, M. Foret, S. Ayrinhac, P. Emery, and B. Rufflé, Phys. Rev. B 77, 100201(R) (2008).

[17] O. Matsuda and O. B. Wright, Anal. Sci. 17, S216 (2001).

[18] P. Lunkenheimer and A. Loidl, Chem. Phys. 284, 205 (2002).

[19] F. Scarponi, L. Comez, D. Fioretto, and L. Palmieri, Phys. Rev. B 70, 054203 (2004).

[20] R. Slayton and K. A. Nelson, J. Chem. Phys. 120, 3919 (2004).

[21] J. Wuttke et al., Phys. Rev. Lett. 72, 3052 (1994).

[22] A. Brodin and E. A. Rössler, Eur. Phys. J. B 44, 3 (2005).

[23] K.F. Herzfeld and T. A. Litovitz, Absorption and Dispersion of Ultrasonic Waves (Academic Press, New York and London, 1959).

[24] C. J. Morath and H. J. Maris, Phys. Rev. B 54, 203 (1996).

[25] David R. Lide, Handbook of Chemistry and Physics (CRC Press, Boca Raton, 2007).

[26] C. Glorieux et al., J. Appl. Phys. 99, 013511 (2006).

[27] S. Granick, Science 253, 1374 (1991).

[28] J. Klein and E. Kumacheva, Science 269, 816 (1995).

[29] M. Heuberger, Science 292, 905 (2001). 